\title{
Optimization Design of Car front Structure Based on Pedestrian Protection
}

\author{
Yao Jia ${ }^{1, a}$, Liu Mingjia ${ }^{2, b}$, Luwei ${ }^{1, c,},{ }^{,}$Luan jiyi ${ }^{1, d}$
}

1College of mechanical engineering, Jiamusi University, China, 154007

2 Ningbo Jili Luoyou Engine Components Co., Ltd, Ningbo, China,315000

ajiahuiyuyao@163.com, b13845459536@163.com, 'lujunqi2001@163.com, ${ }^{\mathrm{d}} 453736626 @ q q . c o m$

*Corresponding author: lujunqi2001@163.com

\section{Keywords: Front structure; Pedestrian protection; Optimization design; Simulation analysis}

Abstract. For reducing the injuries of pedestrians when the collision accidents between pedestrian and vehicle occur, the design of car front structure has been proposed based on pedestrian protection, the design scheme includes a mechanical cushion structure of car bumper and a mechanical bounce device of hood cover triggered by outer-force. CREO software has been used for the establishment of design model, static analysis and structural analysis have been carried under the simulate environment, also the effectiveness of the design scheme for pedestrians protection has been verified.

\section{Introduction}

Among so frequent traffic accidents, pedestrians as the vulnerable groups are most likely injured[1]. In road accidents, heads and legs of pedestrians have a great possibility of being damaged, which accounts for $31.70 \%, 28.80 \%$ respectively, head and leg injuries in traffic accidents are the main damages suffered by pedestrians[2].

Researches of head and leg protection have been developed by traffic safety researchers worldwide, a lot of researches showed: the structural parameters of the bumper can have a very significant impact on the lower extremity injuries, changing the structure parameters of the bumper to make the lower limb of pedestrian enduring even force, can significantly reduce the damage degree; the head of a pedestrian normally comes into contact with the hood or windshield, the inclination angle of the hood or windshield has a particular big impact on the head injury[3]. For the hood, the angle of inclination can be appropriately increased to shorten the pedestrian-car collision time, while increasing the distance between the rigid parts and the engine compartment, and increasing the deformation space of the hood, both can reduce pedestrian injuries.

In previous studies, most researches have been carried considering only the heads protection or the lower extremities protection[4]. In this paper, the bumper structure has energy absorbing ability, and the engine front cover part has a mechanical design to achieve a change of the tilt angle of engine hood, also taking into account the energy absorption design. Comprehensive protection of the two has great practical significance for the safety of pedestrians and car manufacturers to perform pedestrian protection tests. A light energy absorbing material has been chosen as the sandwich layer of the bumper and hood, wherein the rubber column energy absorbing material using rubber plant fiber composites, its performance both has the strength of fiber and the toughness of rubber. 


\section{Optimal design of mechanical structure of car front}

The device can achieve the dual protection effect, the first can alleviate pedestrian impact force by adjusting the assembly when the pedestrian and vehicle impact force is not heavy; the second can adjust the inclination angle of the engine cover body, by appropriately shortening the collision time of pedestrian and automobile, meanwhile can increase the distance of the rigidity compartment of the engine cover increasing the deformation space, to achieve the goal of mitigating pedestrian injuries through the trigger lever and the interaction between the cam mechanism and the lifting mechanism, the function can be obtained when the impact force is beyond the maximum elasticity force endured by the front elastic regulating assembly.

Structure design. The main parts of the active engine hood are the trigger lever, cam mechanism, lifting devices, hood and hood hinges besides bumper and floor part, shown in Fig.1. When Front bumper endures impact force, pushing the trigger lever rearward movement, as well as changing the transfer direction of the force and increasing the force through the cam mechanism, starting the lifting devices, lifting the hood cover under the action of the spring, the lift distance can be controlled by the limit role of the engine hinge, the system work together and can protect pedestrians better.

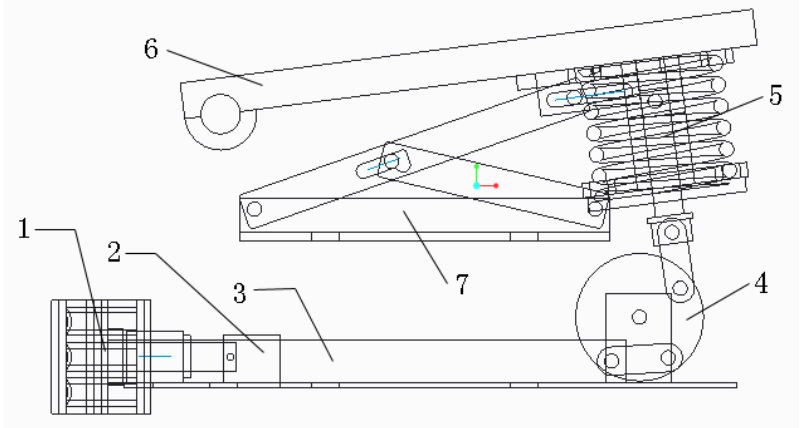

1-bumper 2- floor 3-the trigger lever 4-cam mechanism 5-hood lifting devices 6-hood 7- hinge Fig. 1 Structure of active engine hood

Three-dimensional modeling. As shown in Fig.2, the external bumper has a thin crust, which is stamped from a thin steel plate, when impact occurring, the bumper will quickly deformed and wrinkled, the energy can be absorbed partly, reduce the pedestrian collision damage; there is a very effective internal energy absorbing structure, which is made of rubber plant fiber composite layer of cylindrical ordered, so can ensure the uniformity of force. The additional springs can also have a good energy-absorbing effect. In the event of a collision, the outer sheet of bumper will first format wrinkles, absorb some energy, followed by an internal rubber plant fiber composite cushion layer absorbing energy, if incomplete, the additional strong buffer springs, buffering and fully guaranteeing the safety of pedestrians. 


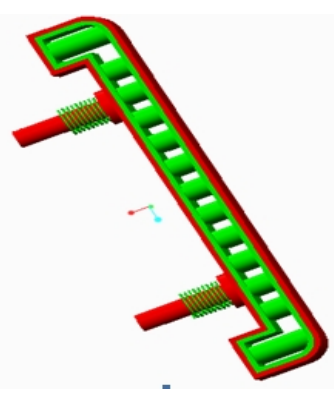

Fig.2 Energy-absorbing bumper of rubber-fiber composite layer

As in Fig.3, the active hood is adopted pure mechanical driving structure. Hood cover is also designed as a sandwich structure, the inner material selection is the same as the bumper. The external hood has a thin steel crust, cylindrical rubber plant fiber composite layer is ordered in the inner part. The trigger lever, cam mechanism, lifting device and hood hinges parts work together to obtain the lifting motion and adjust the inclination angle of the hood cover. This pure mechanical lifting hood need no sensors to collect information, this design is more suitable for vans, trucks and other diesel engines cars, thus pedestrian protection is more direct and effective with low cost mechanical design.

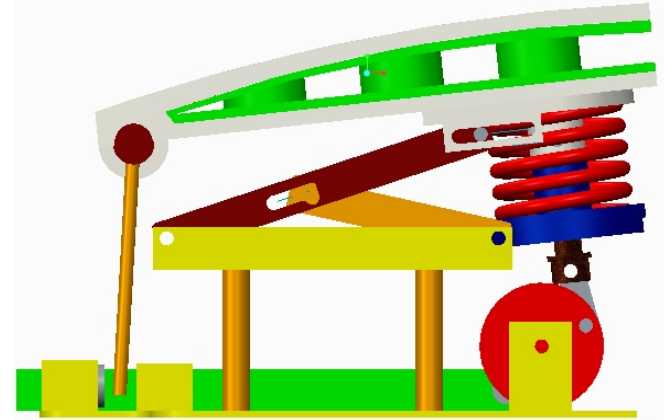

Fig.3 Lifting energy-absorb hood

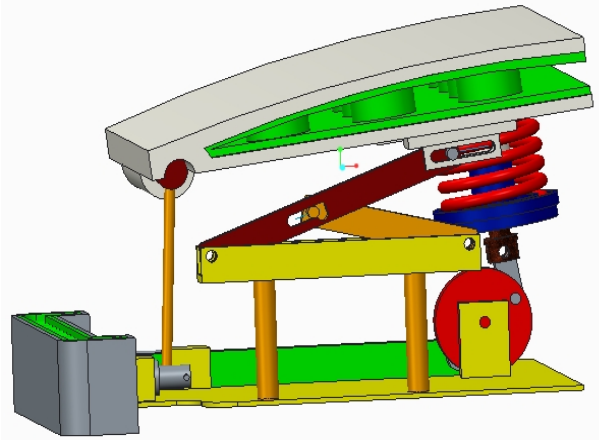

Fig.4 Total model of car front structure

The final design (as shown in Fig.4) can be concluded as the combination of the spring rubber bumper and the energy absorbing lifting hood. The bumper can absorb energy and reduce pedestrian leg injuries; the hood can lift when impacts occur,thus reduce pedestrian head injuries through the energy-absorbing and speed-decreasing.

\section{Simulate static analysis of the car front structure}

When pedestrian accidents occur, the leg of the pedestrian firstly contact with the front bumper, followed by the upper body of pedestrians contact with the hood cover. To analyze the collision and the deformation of the bumper and the hood, the collision area was chosen as the central collision region, twice the size of the width of the pedestrian body. The following study was done (frontal impact) at a special collision by using simulation analysis[3].

Selection of the material and collision force parameters. For the bumper and hood material selection, taking into account the cost and energy-absorbing effect, sheet steel was selected as external package of car bumpers and hood; A new plant fiber mixed rubber cushioning material was selected as the inner energy absorbing material .

Car-pedestrian collision accidents occurred mainly in the speed of $40 \sim 50 \mathrm{~km} / \mathrm{h} \mathrm{[5]}$, supposing the vehicle weight 2 tons, so the force was estimated as $30000 \mathrm{~N}$. The deformation of the hood and bumper was observed under this condition. 
Static simulate analysis of bumper. Using the 'Simulate' function to perform the static pressure test of bumpers, the stress and deformation of the bumper is shown in Fig.5. Fig.5(a) is a stress deformation map, from the deformed plot, the bumper produce stress deformation when a pedestrian collision occurs. There is a huge amount of deformation in the impact zone, the internal rubber plant fiber composite layer is substantially crushed, the bumper in the event of a collision has a large deformation, but due to the efficient cushioning structure with its good energy absorption effect, conducive to better protect the safety of pedestrians legs.

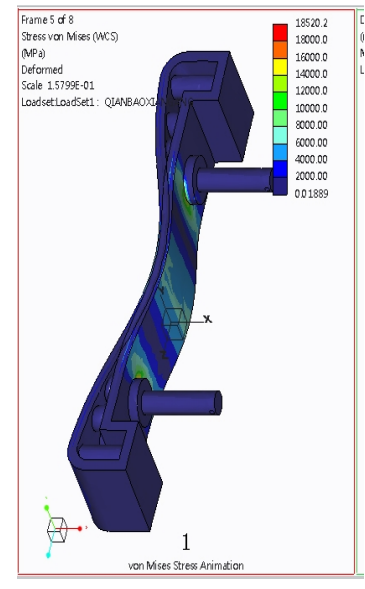

(a) stress map

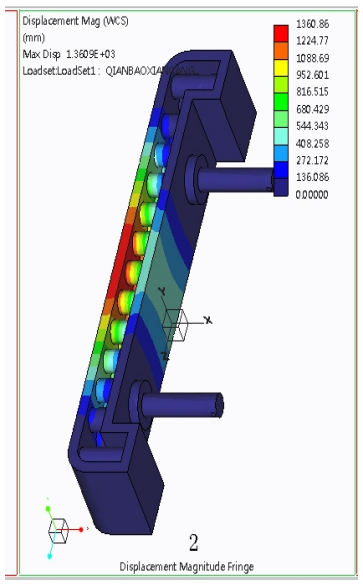

(b) displacement deformation map

Fig.5 Static simulate analysis of bumper

Fig. 5(b), as the displacement map of the bumper shows that when subjects to external impact, the deformation amount of the contact area is great, it was found that there is an excellent energy absorption effect from the joint use of the spring and the cushioning material, so the leg of the pedestrian has good protection. And no substantially deformation of the inner plate can be detected, so in the event of a collision, the internal drive mechanism can also be operated normally, the hood cover can remain the lifting function so the protective effect remains for the pedestrians.

Static analysis of car hood.

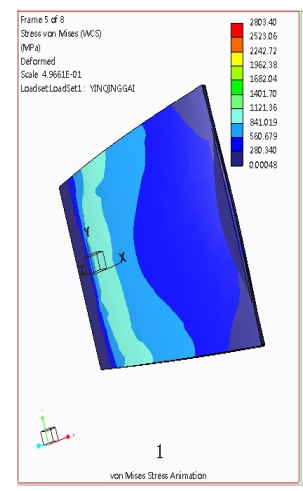

(a) stress map

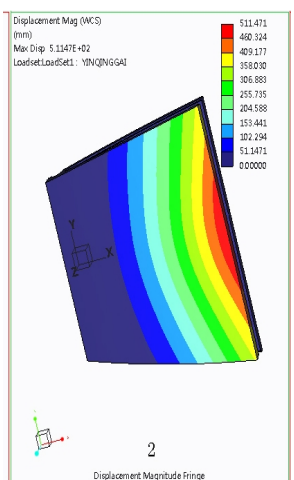

(b) displacement deformation map

Fig.6 static analysis of hood simulation

Using the 'Simulate' function to perform the static pressure test of hood cover[6], the stress and deformation of the hood cover as shown in Fig. 6. Fig.6(a) and (b) are the stress map and displacement deformation map of hood cover. From the deformed plot, it was found that the evident deformation occurred in the zone near the windshield, and the main pedestrian crash site is just there, modal figure shows the deformation is more obvious, it can be understood as the hood has pedestrians protection effect significantly, suitable for installing on cars. And almost no 
deformation of the inner plate can be observed, which can ensure that the internal hinge and lifting devices also operating normally in the event of a collision, so the better protective effect of the hood can be verified.

\section{Conclusions}

The design purpose is for the pedestrian protection, the optimal solution with the combination of energy-absorbing bumper and active hood form the final design of the car frontal structure. When an accident occurs, the legs of pedestrians firstly contacted with car bumper, the car's energy-absorbing bumper structure play a protection role, a part of the collision energy can be absorbed; then causing the lift of the hood cover, which can shorten the collision distance while preventing the contact of the pedestrians with the lower edge of the front windshield, the mechanical structure design and the cushioning design of hood cover can also play a role in preventing the occurrence of serious pedestrian head injuries. The results of the finite element analysis can prove the effectiveness of the design. Further improve the functionality of the system to provide better guarantees for the safety of pedestrians can be expected.

\section{Acknowledgements}

This project was supported by the Youth backbone support plan of Heilongjiang universities(1254G059) .

\section{References}

[1] Z. Xiao, C.H. Yu, F.H. Mo, M. Du and F. Li, Design of Energy Absorber for Front Bumper with Concurrent Considerations of Pedestrian Protection and Low-Speed Collision, Automotive Engineering. 03 (2016) 296-301.

[2] L.W. Sun, M.N. Xie, Y.K. Yang, An Analysis Based on a Modal of Pedestrian Head Collision Protection Simulaation. Journal of Liaoning Provincial College of Communications, 05 (2015) 23-28.

[3] Y.Y. Gu, Simulation and Analysis of Vehicle Bumper Based on the Pedestrian Lower Leg Protection, Wuhan University of Technology, (2013).

[4] C.Q. Zhang, J.L. Pan. Crash Safety Research of a Passenger Car Based on Pedestrian Protection, Agricultural Equipment \& Vehicle Engineering, 09 (2013) 35-38.

[5] S. Oikawa,Y. Matsui,T. Sakurai. Characteristics of collision damage mitigation braking system for pedestrian protection. International Journal of Automotive Technology, (2014) 157.

[6] Shunsuke Takagi, Koki Tai, Asato Wakabayashi, Yasuhiro Matsui, Validation of restoration time for pedestrian headform impactor skin, International Journal of Crashworthiness, (2013) 186. 